\title{
economics
}

Rationing problems with payoff thresholds

\section{Pere Timoner}

Josep Maria Izquierdo 


\title{
Rationing problems with payoff thresholds
}

\begin{abstract}
An extension of the standard rationing model is introduced. Agents are not only identi fied by their respective claims over some amount of a scarce resource, but also by some payoff thresholds. These thresholds introduce exogenous differences among agents (full or partial priority, past allocations, past debts, ...) that may influence the final distribution. Within this framework we provide generalizations of the constrained equal awards rule and the constrained equal losses rule. We show that these generalized rules are dual from each other. We characterize the generalization of the equal awards rule by using the properties of consistency, path-independence and compensated exemption. Finally, we use the duality between rules to characterize the generalization of the equal losses solution.
\end{abstract}

JEL Codes: D63.

Keywords: Rationing, equal awards rule, equal losses rule, claims problems.

\author{
Pere Timoner \\ Facultat d'Economia i Empresa \\ Universitat de Barcelona \\ Josep Maria Izquierdo \\ Facultat d'Economia i Empresa \\ Universitat de Barcelona
}

Acknowledgements: We thank Carles Rafels for his helpful comments. The authors acknowledge the support by research grant ECO2011-22765 (Spanish Ministry of Science and Innovation and FEDER) and 2009SGR960 (Government of Catalonia). The first author also acknowledges program APIF (University of Barcelona).

ISSN 1136-8365 


\section{Introduction}

A standard rationing problem is an allocation problem, where each agent $i$ of a given set $N$ claims a quantity $c_{i}$ over an amount $r$ of some (perfectly divisible) resource (e.g., money) that is insufficient to satisfy all the claims, $\sum_{i \in N} c_{i} \geq r$. A solution to this problem assigns to each agent $i \in N$ a part $x_{i}$ of the available amount of resource not larger than his claim. Assignment taxes, bankruptcy and distribution of emergency supplies are examples of rationing problems.

Standard rationing problems have been widely studied in the literature (see the survey of Thomson, 2003). In the classical approach agents are represented by one characteristic, the claimed amount, and they are rewarded according to it. Since ancient times, several solutions to this problem have been proposed and analysed (see O'Neill, 1982 and Aumann and Maschler, 1985). Among them we highlight the constrained equal awards rule $(C E A)$ that equalizes gains $\left(x_{i}=\min \left\{c_{i}, \lambda\right\}\right)$, the constrained equal losses rule $(C E L)$ that equalizes losses from claims $\left(x_{i}=\max \left\{0, c_{i}-\lambda\right\}\right)$ or the proportional rule that equalizes average rewards with respect to claims $\left(x_{i}=\lambda c_{i}\right)$, where the parameter $\lambda$ that appears in the formulas represents the individual gain, the individual loss and the per unit gain assigned to each agent, respectively.

Standard rationing analysis consider that claims are the only relevant information that affects the final distribution. Recently, several authors study complex rationing situations, where not only claims, but also individual rights or other entitlements affect the final distribution. Pulido et al. (2002 and 2008) and Hougaard et al. (2012, 2013a and 2013b) introduce references or baselines based on past experience or exogenous entitlements in order to refine the claims of agents.

When applying the constrained equal awards rule, the final distribution can be viewed as a dynamic allocation process where agents are assigned additional equal quantities of the scarce resource at the same speed until the total available amount is fully distributed. However, exogenous arguments might recommend assigning no payoff to an agent until another agent would 
have received a minimum amount of resource. To incorporate this fact in the model we consider different payoff thresholds from where agents start to receive payoff.

In order to illustrate the concept of payoff threshold let us consider the following examples. Imagine that every year different departments of a university claim funds over a limited budget and the latest year one of the departments has received an extra assignment to renovate its offices. It seems plausible that this extra assignment influences the current assignment, so that a positive payoff threshold is fixed for this department for the present and maybe for future allocations. Continuing with the same example, maybe the university had promised some amount of money to another department that it has not been finally satisfied. Then, in the current allocation it also seems plausible that this department starts receiving funds before other departments do. This fact will be included in our model by assigning a negative payoff threshold to this department representing a debt to it. Obviously a situation may combine positive thresholds corresponding to some agents and negative ones corresponding to others.

Another example is food supplies to refugees. Consider that the food distributor divides the refugees of a camp in three categories; children, adults and elders, fully prioritizing the first category over the others which means that adults and elders will not receive anything until each child receives all his claim. This fact can be introduced in our model by assigning to each adult and to each elder a payoff threshold equal to the highest claim among children. Furthermore, imagine now that the elders have, at his turn, partial priority over the adults, which means that the adults will not receive anything until each elder had receive a minimum amount (e.g., a subsistence level). To incorporate this fact, we will add to the threshold of adults this minimum amount. In this way, payoff thresholds can partially prioritize some agents with respect to others. Partial priority may also arises from a rationing situation where there are differences in the wealth of agents; including payoff thresholds can help to discriminate among them. At this point let us remark 
that asymmetric allocations were already analysed in Moulin (2000). This author assigns weights to agents and distributes proportionally with respect to the weights (up to the claims) awards and losses. He also combines these weighted solutions with full priority rules. In our approach the asymmetries are included in the definition of the problem and the rules that we apply, preserve the idea of equal distribution.

Another interesting example can be a periodical allocation problem within the same set of agents where each agent makes in each period an estimation of his claim. If the claim estimation of an agent has been higher than his real needs, receiving an excessive assignment for that reason, then it seems plausible that we fix a positive payoff threshold for this agent in the next period. Finally, in a multiple bankruptcy situation where agents claim for different types of assets (buildings, equities, cash, ...), it is reasonable to think that the allocation of an asset should be influenced by the amount received in the rest of assets.

In Section 2 we introduce the main notations, we define a rationing problem with payoff thresholds and we extend the constrained equal awards rule and the constrained equal losses rule to new framework. In Section 3 we characterize axiomatically both rules by extending, and in some cases, adapting the axioms used to characterize the rules in the standard rationing context (Herrero and Villar, 2001). In Section 4 we conclude.

\section{Rationing problems with payoff thresholds and rules}

Let $\mathbb{N}$ denote the set of all potential agents (a set with an infinite number of members), and let $\mathcal{N}$ be the family of all finite subsets of $\mathbb{N}$.

Given a finite subset of agents $N=\{1,2, \ldots, n\} \in \mathcal{N}$, a standard rationing problem is to distribute $r \geq 0$ among these $n$ agents with claims $c=\left(c_{1}, c_{2}, \ldots, c_{n}\right) \in \mathbb{R}_{+}^{N}$. It is assumed that $r \leq \sum_{i \in N} c_{i}$ since otherwise no rationing problem exists. We denote a standard rationing problem by the 
pair $(r, c) \in \mathbb{R}_{+} \times \mathbb{R}_{+}^{N}$.

An allocation of $r$ is represented by a vector $x=\left(x_{1}, x_{2}, \ldots, x_{n}\right) \in \mathbb{R}^{N}$ such that $0 \leq x_{i} \leq c_{i}$ and $\sum_{i \in N} x_{i}=r$, where $x_{i}$ represents the payoff associated to agent $i \in N$. Given a vector $x \in \mathbb{R}^{N}$ and a subset $S \subseteq N$, we denote by $x_{\mid S} \in \mathbb{R}^{S}$ the vector $x$ restricted to the members of $S$. Finally, for all $S \subseteq N$, we denote by $s$ the cardinality of $S$. A rationing rule associates to each rationing problem a unique allocation.

Next we define the $C E A$ and the $C E L$ rules.

Definition 1. (Constrained equal awards rule, $C E A$ ). For any rationing problem $(r, c) \in \mathbb{R}_{+} \times \mathbb{R}_{+}^{N}$ the $C E A$ rule is defined as

$$
C E A_{i}(r, c)=\min \left\{c_{i}, \lambda\right\}, \text { for all } i \in N
$$

where $\lambda \in \mathbb{R}_{+}$satisfies $\sum_{i \in N} \min \left\{c_{i}, \lambda\right\}=r$.

Definition 2. (Constrained equal losses rule, $C E L)$. For any rationing problem $(r, c) \in \mathbb{R}_{+} \times \mathbb{R}_{+}^{N}$ the $C E L$ rule is defined as

$$
C E L_{i}(r, c)=\max \left\{0, c_{i}-\lambda\right\}, \text { for all } i \in N
$$

where $\lambda \in \mathbb{R}_{+}$satisfies $\sum_{i \in N} \max \left\{0, c_{i}-\lambda\right\}=r$.

Now suppose that each agent has been assigned a payoff threshold. In order to illustrate this new concept, let us discuss the following example that aims to extend the $C E A$ rule to new framework.

Example 1. Consider the following four-person standard rationing problem (without payoff thresholds):

$$
r=9 \text { and }\left(c_{1}, c_{2}, c_{3}, c_{4}\right)=(2.5,1.5,2,4.5) .
$$

If we apply the $C E A$ rule to this problem we obtain the allocation $\left(x_{1}, x_{2}, x_{3}, x_{4}\right)$ $=(2.5,1.5,2,3)$ that can be obtained in several steps by assigning progressively equal additional units of payoff: first, by assigning 1.5 units to each agent (in this case agent 2 is fully satisfied and leaves the picture); second, 
the assignment process follows by additionally assigning 0.5 units to agents 1,3 and 4 (in this case agent 3 is fully satisfied and also leaves the picture); third, we further assign 0.5 units to agents 1 and 4 (and agent 1 is also fully satisfied); finally the procedure ends by assigning the remaining 0.5 units to agent 4 .

Let us add to the problem the following vector of payoff thresholds:

$$
\left(\delta_{1}, \delta_{2}, \delta_{3}, \delta_{4}\right)=(0,1,2,-1)
$$

Recall that the positive payoff thresholds of agents 1, 2 and 3 might be interpreted as a consequence of earlier allocations to these agents, while the negative threshold of agent 4 might be interpreted as a debt to this agent in the past. Let us apply now the same equalizing procedure. Notice that, since there is a debt to agent 4, we will first assign 1 unit to this agent. In the next step, we will continue equally assigning to agents 1 and 4 but not to agents 2 and 3, since they have a positive payoff threshold; in particular agent 2 (and so agent 3) will not receive any payoff until agents 1 and 4 had reach a net payoff (of debts) of 1 unit (the payoff threshold of agent 2). Then agents 1, 2 and 4 continue receiving payoff until they have been assigned a net payoff of 2 units (the payoff threshold of agent 3). Then, all agents additionally receive 0.5 units each until agents 1 and 2 are fully satisfied (and they leave the picture). Finally, the remaining unit left is split equally between agents 3 and 4. The final allocation is $x=(2.5,1.5,1,4)$. A graphic representation of this procedure can be found in Figure 1 which is inspired in the hydraulic representation of rationing rules given by Kaminski (2000).

The aim of a rationing problem with payoff thresholds is to distribute an amount of a scarce resource in a fair way but taking into account the payoff thresholds.

Definition 3. A rationing problem with payoff thresholds is a triple $(r, c, \delta)$, where $r \in \mathbb{R}_{+}$is the amount of resource, $c \in \mathbb{R}_{+}^{N}$ is the vector of claims such that $r \leq \sum_{i \in N} c_{i}$ and $\delta \in \mathbb{R}^{N}$ is the vector of payoff thresholds. 


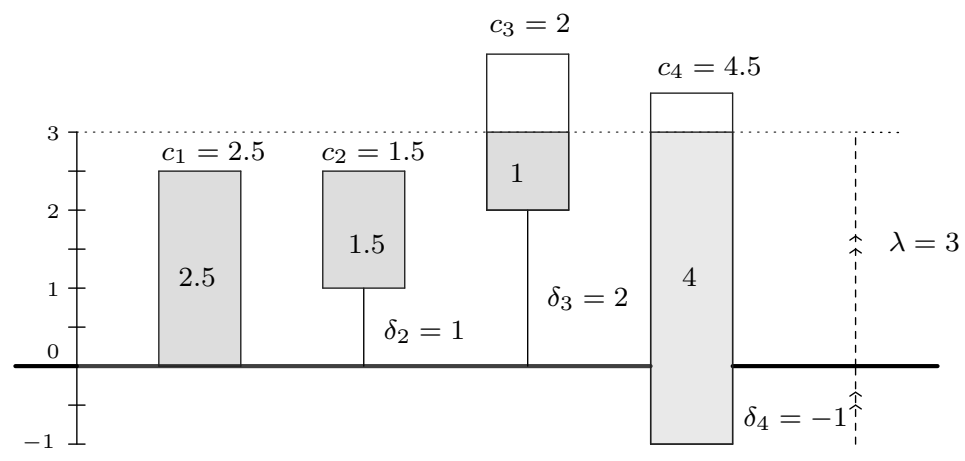

Figure 1: Equalizing awards with payoff thresholds.

We denote by $\mathcal{R}^{N}$ the set of all rationing problems with payoff thresholds and agent's set $N$. The definition of an allocation rule for these problems does not differ essentially from the standard definition.

Definition 4. A generalized rationing rule is a function $F$ that associates to each rationing problem with payoff thresholds $(r, c, \delta) \in \mathcal{R}^{N}$ a unique allocation $x^{*}=F(r, c, \delta) \in \mathbb{R}_{+}^{N}$ such that

- $\sum_{i \in N} x_{i}^{*}=r$ (efficiency) and

- $0 \leq x_{i}^{*} \leq c_{i}$, for all $i \in N$.

Next we will extend the constrained equal awards rule to new framework.

Definition 5. (Generalized equal awards rule, GEA). For any rationing problem with payoff thresholds $(r, c, \delta) \in \mathcal{R}^{N}$ the $G E A$ rule is defined as ${ }^{1}$

$$
G E A_{i}(r, c, \delta)=\min \left\{c_{i},\left(\lambda-\delta_{i}\right)_{+}\right\}, \text {for all } i \in N
$$

where $\lambda \in \mathbb{R}$ satisfies that $\sum_{i \in N} G E A_{i}(r, c, \delta)=r$.

\footnotetext{
${ }^{1}$ From now we will use the following notation: for all $a \in \mathbb{R},(a)_{+}=\max \{0, a\}$.
} 
Notice that the $G E A$ rule is well-defined. Indeed, by Bolzano's Theorem applied to the continuous function $\varphi(\lambda)=\sum_{i \in N} \varphi_{i}(\lambda)=\sum_{i \in N} \min \left\{c_{i},\left(\lambda-\delta_{i}\right)_{+}\right\}$, the existence of a value $\lambda$ such that $\varphi(\lambda)=r$ is guaranteed since $\varphi\left(\min _{i \in N}\left\{\delta_{i}\right\}\right)$ $\leq r \leq \varphi\left(\max _{i \in N}\left\{c_{i}+\delta_{i}\right\}\right)$. Moreover, let us suppose that there would exist $\lambda, \lambda^{\prime} \in \mathbb{R}$, with $\lambda<\lambda^{\prime}$, such that $\varphi(\lambda)=\varphi\left(\lambda^{\prime}\right)=r$. As the reader may check $\varphi_{k}(\lambda)$ is a non-decreasing function for all $k \in N$. Hence, we have that $\varphi_{k}(\lambda) \leq \varphi_{k}\left(\lambda^{\prime}\right)$, for all $k \in N$. Therefore, we get $\sum_{k \in N} \varphi_{k}(\lambda) \leq$ $\sum_{k \in N} \varphi_{k}\left(\lambda^{\prime}\right)=r$ and so $\varphi_{k}(\lambda)=\varphi_{k}\left(\lambda^{\prime}\right)$ for all $k \in N$. We conclude that the solution is unique and so it is well-defined for all problem.

Obviously the $G E A$ rule coincides with the $C E A$ rule for standard rationing problems (without payoff thresholds). In fact they coincide if payoff thresholds of agents are all equal.

Remark 1. If $\delta=(\alpha, \alpha, \ldots, \alpha) \in \mathbb{R}^{N}$, then $G E A(r, c, \delta)=C E A(r, c)$.

The $G E A$ rule can also be described by means of comparing the payoffs for any pair of agents.

Proposition 1. Let $(r, c, \delta)$ be a rationing problem with payoff thresholds and let $x^{*}=F(r, c, \delta)$. Then,

$$
\begin{aligned}
x^{*}=G E A(r, c, \delta) \Leftrightarrow & \text { for all } i, j \in N, \text { such that } x_{i}^{*}+\delta_{i}<x_{j}^{*}+\delta_{j}, \\
& \text { it holds that either } x_{j}^{*}=0 \text {, or } x_{i}^{*}=c_{i} .
\end{aligned}
$$

Proof. $\Rightarrow)$ Let us suppose that $x^{*}=G E A(r, c, \delta)$ and there exist $i, j \in N$, such that $x_{i}^{*}+\delta_{i}<x_{j}^{*}+\delta_{j}$ but $x_{j}^{*}>0$ and $x_{i}^{*}<c_{i}$. Hence, $x_{i}^{*}=\left(\lambda-\delta_{i}\right)_{+}$, $\lambda-\delta_{j}>0$, and so

$$
\begin{aligned}
x_{i}^{*}+\delta_{i} & =\left(\lambda-\delta_{i}\right)_{+}+\delta_{i} \geq \lambda \geq \min \left\{c_{j}+\delta_{j}, \lambda\right\} \\
& =\min \left\{c_{j}, \lambda-\delta_{j}\right\}+\delta_{j}=\min \left\{c_{j},\left(\lambda-\delta_{j}\right)_{+}\right\}+\delta_{j}=x_{j}^{*}+\delta_{j} .
\end{aligned}
$$

Hence, we reach a contradiction with the hypothesis $x_{i}^{*}+\delta_{i}<x_{j}^{*}+\delta_{j}$ and we conclude that either $x_{i}^{*}=c_{i}$, or $x_{j}^{*}=0$. 
$\Leftarrow)$ Let us suppose that for all $i, j \in N$, such that $x_{i}^{*}+\delta_{i}<x_{j}^{*}+\delta_{j}$, it holds that either $x_{j}^{*}=0$, or $x_{i}^{*}=c_{i}$, but $x^{*} \neq G E A(r, c, \delta)$. Then, by efficiency, there exist $i, j \in N$ such that

$$
0 \leq x_{i}^{*}<G E A_{i}(r, c, \delta) \leq c_{i} \text { and } c_{j} \geq x_{j}^{*}>G E A_{j}(r, c, \delta) \geq 0 .
$$

This means that $x_{i}^{*}<c_{i}, \lambda-\delta_{i}>0$ and $\left(\lambda-\delta_{j}\right)_{+}<c_{j}$. However,

$$
\begin{aligned}
x_{j}^{*}+\delta_{j} & >G E A_{j}(r, c, \delta)+\delta_{j}=\left(\lambda-\delta_{j}\right)_{+}+\delta_{j} \geq \lambda \geq \min \left\{c_{i}+\delta_{i}, \lambda\right\} \\
& =\min \left\{c_{i}, \lambda-\delta_{i}\right\}+\delta_{i}=G E A_{i}(r, c, \delta)+\delta_{i}>x_{i}^{*}+\delta_{i} .
\end{aligned}
$$

By assumption, it should hold that either $x_{j}^{*}=0$, or $x_{i}^{*}=c_{i}$, but this contradicts (1). Hence we conclude that $x^{*}=G E A(r, c, \delta)$.

Now we extend the idea of equalizing losses to the payoff thresholds rationing framework. The loss of an agent is the difference between his claim and his assigned payoff. If an agent has a positive payoff threshold, then he can suffer a higher loss compare to an agent without payoff threshold or with a negative payoff threshold (a debt or an acquired loss). We define the generalized equal losses rule as follows:

Definition 6. (Generalized equal losses rule, GEL). For any rationing problem with payoff thresholds $(r, c, \delta) \in \mathcal{R}^{N}$ the $G E L$ rule is defined as

$$
G E L_{i}(r, c, \delta)=\max \left\{0, c_{i}-\left(\lambda+\delta_{i}\right)_{+}\right\}, \text {for all } i \in N,
$$

where $\lambda \in \mathbb{R}$ satisfies that $\sum_{i \in N} G E L_{i}(r, c, \delta)=r$.

The reader may check that the $G E L$ rule is well-defined by using similar arguments as for the case of the $G E A$ rule.

Remark 2. If $\delta=(\alpha, \alpha, \ldots, \alpha) \in \mathbb{R}^{N}$, then $G E L(r, c, \delta)=C E L(r, c)$.

Next example illustrates the idea of the $G E L$ rule. 
Example 2. Consider the following three-person problem with $r=2, c=$ $(2,2,2)$ and $\delta=(0,1,-1)$. Notice that the total loss is $L=c_{1}+c_{2}+c_{3}-r=$ $6-2=4$. We first assign losses to agent 2 who has a positive payoff threshold of 1 unit; we assign him 1 unit of loss. Then, we continue assigning losses to agents 1 and 2 , but not to agent 3 since he has a negative payoff threshold (-1 unit) and we will not assign to him any additional loss until agents 1 and 2 were assigned 1 unit of loss. Hence, we assign 1 unit of loss to agent 1 and 1 unit to agent 2 (additional to the unit previously assigned). Notice that at this point we have distributed a total of 3 units of loss. Moreover, agent 2 has already received a loss of 2 units equal to his claim and, obviously, he will not receive more losses and leaves the picture. Finally, the remaining 1 unit of loss is split equally between agents 1 and 3. The final distribution of losses is $(1.5,2,0.5)$ and so the payoff assigned is $x=(2-1.5,2-2,2-0.5)=$ $(0.5,0,1.5)$ that contrast with the non payoff thresholds case where the final payoff is $x^{\prime}=\left(\frac{2}{3}, \frac{2}{3}, \frac{2}{3}\right)$. A graphic representation of this procedure can be found in Figure 2.

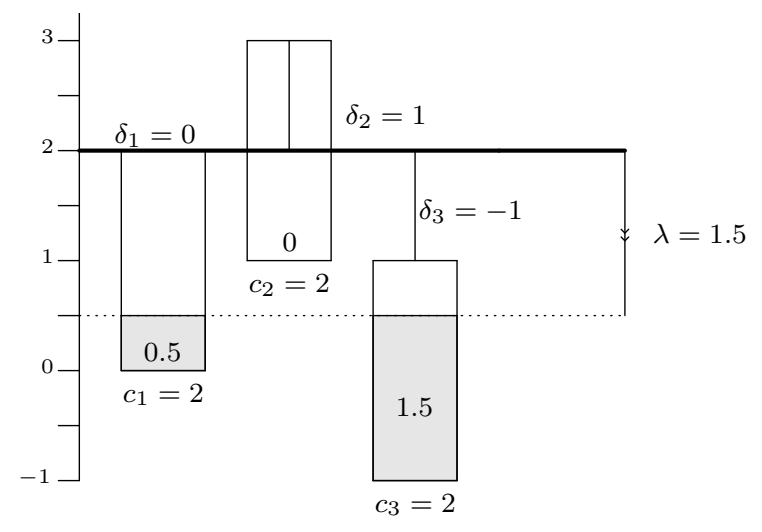

Figure 2: An Example of the GEL rule.

In the standard rationing framework, the $C E A$ rule and the $C E L$ rule are dual rules. This means that one rule distributes the total gain $r$, in the primal problem $(r, c)$, in the same way as the other rule distributes the 
total loss $L$, where $L=\sum_{i \in N} c_{i}-r$, in the dual problem $(L, c)$. The idea of duality can be adapted for rationing problems with payoff thresholds but taking into account that positive payoff thresholds become negative (and vice versa) when passing from the primal problem $(r, c, \delta)$ to the dual problem $(L, c,-\delta)$. Next we define formally dual rules.

Definition 7. $F^{*}$ is the dual rule of $F$ if, for all $(r, c, \delta) \in \mathcal{R}^{N}$,

$$
F^{*}(r, c, \delta)=c-F(L, c,-\delta) .
$$

The duality of the $G E A$ and the $G E L$ rule is maintained as it is the case for the $C E A$ and the $C E L$ rule in the standard framework.

Proposition 2. The GEA and the GEL are dual rules from each other.

Proof. Let us first prove that $G E A(r, c, \delta)=c-G E L(L, c,-\delta)$. By definition and for all $i \in N$,

$$
\begin{aligned}
G E A_{i}(r, c, \delta) & =\min \left\{c_{i},\left(\lambda-\delta_{i}\right)_{+}\right\} \\
& =c_{i}+\min \left\{0,\left(\lambda-\delta_{i}\right)_{+}-c_{i}\right\} \\
& =c_{i}-\max \left\{0, c_{i}-\left(\lambda-\delta_{i}\right)_{+}\right\} .
\end{aligned}
$$

By (2), $\sum_{i \in N} G E A_{i}(r, c, \delta)=\sum_{i \in N} c_{i}-\sum_{i \in N} \max \left\{0, c_{i}-\left(\lambda-\delta_{i}\right)_{+}\right\}$and so $\sum_{i \in N} \max \left\{0, c_{i}-\left(\lambda-\delta_{i}\right)_{+}\right\}=\sum_{i \in N} c_{i}-r=L$. Hence, $\max \left\{0, c_{i}-(\lambda-\right.$ $\left.\left.\delta_{i}\right)_{+}\right\}=G E L_{i}(L, c,-\delta)$ for all $i \in N$.

Next we prove that $G E L(r, c, \delta)=c-G E A(L, c, \delta)$. By definition, for all $i \in N$,

$$
\begin{aligned}
G E L_{i}(r, c, \delta) & =\max \left\{0, c_{i}-\left(\lambda+\delta_{i}\right)_{+}\right\} \\
& =c_{i}-\min \left\{c_{i},\left(\lambda+\delta_{i}\right)_{+}\right\} .
\end{aligned}
$$

By (3), $\sum_{i \in N} G E L_{i}(r, c, \delta)=\sum_{i \in N} c_{i}-\sum_{i \in N} \min \left\{c_{i},\left(\lambda+\delta_{i}\right)_{+}\right\}$and so $\sum_{i \in N} \min \left\{c_{i},\left(\lambda+\delta_{i}\right)_{+}\right\}=\sum_{i \in N} c_{i}-r=L$.

Hence, $\min \left\{c_{i},\left(\lambda+\delta_{i}\right)_{+}\right\}=G E A_{i}(L, c,-\delta)$, for all $i \in N$. We conclude that $G E A$ and $G E L$ are dual rules from each other. 


\section{Axiomatic characterizations}

The $C E A$ and the $C E L$ rule (for standard rationing problems) had been characterized by Herrero and Villar (2001) among others. Besides consistency (a property that is common to most rationing rules) the properties that characterize the $C E A$ rule are path-independence and exemption. In this section we will adapt the characterization to our framework.

First, to characterize the $G E A$ rule we need the properties of pathindependence, consistency and we introduce a new property called compensated exemption that can be viewed as an extension of the classical exemption property for the two-person case.

Path-independence states that if we apply a rule to some problem (obtaining an allocation) but the available amount of resource diminishes suddenly, the new allocation obtained by applying again the rule (with the new amount and the original claims) is equal to the one we would obtain using the previous allocation as claims.

Property 1. A generalized rationing rule $F$ satisfies path-independence if for all $(r, c, \delta) \in \mathcal{R}^{N}$ and $\sum_{i \in N} c_{i} \geq r^{\prime} \geq r$ it holds

$$
F(r, c, \delta)=F\left(r, F\left(r^{\prime}, c, \delta\right), \delta\right)
$$

If a rule satisfies path-independence it is monotonic with respect to the available amount of resource as the reader may check. That is for all $N$, for all $c \in \mathbb{R}^{N}$ and for all $r, r^{\prime}:\left\{r \leq r^{\prime} \leq \sum_{i \in N} c_{i}\right\} \Rightarrow\left\{F(r, c, \delta) \leq F\left(r^{\prime}, c, \delta\right)\right\}$. This property is called resource monotonicity.

Concerning exemption, this property requires that an agent with a small enough claim will not suffer from rationing. In particular the two-person case $N=\{i, j\}$ states that if $c_{i} \leq \frac{r}{2}$ then $x_{i}=c_{i}$.

Compensated exemption is defined just for the two-person case and requires that exemption applies after payoff compensations between agents due to differences in payoff thresholds are carried out. In case of $\delta_{i}>\delta_{j}$, this property requires that if $c_{i} \leq \frac{r-\left(\delta_{i}-\delta_{j}\right)}{2}$ then $x_{i}=c_{i}$. This property reads as 
follows: we apply exemption taking into account not $r$ but the net amount after compensating agent $j$ for the difference between payoff thresholds.

To cover the case where $\delta_{i}<\delta_{j}$, we define in general this property as follows ${ }^{2}$ :

Property 2. A generalized rationing rule $F$ satisfies compensated exemption if for any two-person rationing problem $(r, c, \delta) \in \mathcal{R}^{\{i, j\}}$ it holds that

$$
\text { if } \min \left\{r, c_{i}\right\} \leq \frac{r-\left(\delta_{i}-\delta_{j}\right)}{2} \Longrightarrow F_{i}(r, c, \delta)=\min \left\{r, c_{i}\right\} .
$$

On the other hand, consistency is a property that requires that if we re-evaluate the allocation of resources within a subgroup of agents using the same rule, the allocation does not change.

Property 3. A generalized rationing rule $F$ is consistent if for all $(r, c, \delta) \in$ $\mathcal{R}^{N}$ and all $T \subseteq N$ it holds

$$
F(r, c, \delta)_{\mid T}=F\left(r-\sum_{i \in N \backslash T} F_{i}(r, c, \delta), c_{\mid T}, \delta_{\mid T}\right) .
$$

Next we show that the $G E A$ rule satisfies these properties.

Proposition 3. The GEA rule satisfies path-independence.

Proof. Notice that if $r=r^{\prime}$ the result is straightforward. Let us suppose now that $r<r^{\prime}$. We claim

$$
G E A(r, c, \delta)=G E A\left(r, G E A\left(r^{\prime}, c, \delta\right), \delta\right) .
$$

Let $i \in N$; by definition we have

$$
\begin{aligned}
& G E A_{i}(r, c, \delta)=\min \left\{c_{i},\left(\lambda-\delta_{i}\right)_{+}\right\} \text {with } \sum_{k \in N} G E A_{k}(r, c, \delta)=r, \\
& G E A_{i}\left(r^{\prime}, c, \delta\right)=\min \left\{c_{i},\left(\lambda^{\prime}-\delta_{i}\right)_{+}\right\} \text {with } \sum_{k \in N} G E A_{k}\left(r^{\prime}, c, \delta\right)=r^{\prime} \text { and } \\
& G E A_{i}\left(r, G E A\left(r^{\prime}, c, \delta\right), \delta\right)=\min \left\{\min \left\{c_{i},\left(\lambda^{\prime}-\delta_{i}\right)_{+}\right\},\left(\lambda^{\prime \prime}-\delta_{i}\right)_{+}\right\} \\
& \text {with } \sum_{k \in N} G E A_{k}\left(r, G E A\left(r^{\prime}, c, \delta\right), \delta\right)=r .
\end{aligned}
$$

\footnotetext{
${ }^{2}$ Notice that if $\delta_{i}=\delta_{j}$ then compensated exemption implies exemption for two-person case.
} 
First, we claim

$$
\lambda<\lambda^{\prime}
$$

If not, $\lambda \geq \lambda^{\prime}$, we would have that, for all $i \in N$,

$$
G E A_{i}(r, c, \delta)=\min \left\{c_{i},\left(\lambda-\delta_{i}\right)_{+}\right\} \geq \min \left\{c_{i},\left(\lambda^{\prime}-\delta_{i}\right)_{+}\right\}=G E A_{i}\left(r^{\prime}, c, \delta\right),
$$

and adding up all the above inequalities, we obtain $r=\sum_{i \in N} G E A_{i}(r, c, \delta) \geq$ $\sum_{i \in N} G E A_{i}\left(r^{\prime}, c, \delta\right)=r^{\prime}$ which contradicts $r<r^{\prime}$.

Let us suppose now that $G E A(r, c, \delta) \neq G E A\left(r, G E A\left(r^{\prime}, c, \delta\right), \delta\right)$ and so there exists $i^{*} \in N$ such that

$$
\begin{aligned}
G E A_{i^{*}}(r, c, \delta) & =\min \left\{c_{i^{*}},\left(\lambda-\delta_{i^{*}}\right)_{+}\right\} \\
& <\min \left\{\min \left\{c_{i^{*}},\left(\lambda^{\prime}-\delta_{i^{*}}\right)_{+}\right\},\left(\lambda^{\prime \prime}-\delta_{i^{*}}\right)_{+}\right\} \\
& =G E A_{i^{*}}\left(r, G E A\left(r^{\prime}, c, \delta\right), \delta\right) \leq G E A_{i^{*}}\left(r^{\prime}, c, \delta\right) \leq c_{i^{*}} .
\end{aligned}
$$

Hence, notice that $\min \left\{c_{i^{*}},\left(\lambda-\delta_{i^{*}}\right)_{+}\right\}=\left(\lambda-\delta_{i^{*}}\right)_{+}$. Taking this into account, and substituting in (5), we have

$$
\begin{aligned}
\left(\lambda-\delta_{i^{*}}\right)_{+} & <\min \left\{\min \left\{c_{i^{*}},\left(\lambda^{\prime}-\delta_{i^{*}}\right)_{+}\right\},\left(\lambda^{\prime \prime}-\delta_{i^{*}}\right)_{+}\right\} \\
& \leq\left(\lambda^{\prime \prime}-\delta_{i^{*}}\right)_{+}
\end{aligned}
$$

and so we obtain that $\lambda-\delta_{i^{*}} \leq\left(\lambda-\delta_{i^{*}}\right)_{+}<\left(\lambda^{\prime \prime}-\delta_{i^{*}}\right)_{+}=\lambda^{\prime \prime}-\delta_{i^{*}}$ which implies

$$
\lambda<\lambda^{\prime \prime} .
$$

Moreover, by (4) and (6) we get that, for all $j \in N \backslash\left\{i^{*}\right\}$,

$$
\begin{aligned}
G E A_{j}(r, c, \delta) & =\min \left\{c_{j},\left(\lambda-\delta_{j}\right)_{+}\right\} \\
& \leq \min \left\{c_{j}, \min \left\{\left(\lambda^{\prime}-\delta_{j}\right)_{+},\left(\lambda^{\prime \prime}-\delta_{j}\right)_{+}\right\}\right\} \\
& =\min \left\{\min \left\{c_{j},\left(\lambda^{\prime}-\delta_{j}\right)_{+}\right\},\left(\lambda^{\prime \prime}-\delta_{j}\right)_{+}\right\} \\
& =G E A_{j}\left(r, G E A\left(r^{\prime}, c, \delta\right), \delta\right) .
\end{aligned}
$$


Finally, taking this into account, we conclude that

$$
\begin{aligned}
r & =\sum_{j \in N} G E A_{j}(r, c, \delta)=G E A_{i^{*}}(r, c, \delta)+\sum_{j \in N \backslash\left\{i^{*}\right\}} G E A_{j}(r, c, \delta) \\
& <G E A_{i^{*}}\left(r, G E A\left(r^{\prime}, c, \delta\right), \delta\right)+\sum_{j \in N \backslash\left\{i^{*}\right\}} G E A_{j}(r, c, \delta) \\
& \leq \sum_{j \in N} G E A_{j}\left(r, G E A\left(r^{\prime}, c, \delta\right), \delta\right)=r
\end{aligned}
$$

getting a contradiction. Therefore, we conclude

$$
G E A(r, c, \delta)=G E A\left(r, G E A\left(r^{\prime}, c, \delta\right), \delta\right)
$$

Proposition 4. The GEA rule satisfies compensated exemption.

Proof. Notice that if $r=0$ the result is straightforward. Let $(r, c, \delta) \in$ $\mathcal{R}^{\{i, j\}}$ be a two-person rationing problem with payoff thresholds and let $x^{*}=$ $G E A(r, c, \delta)$. Let us suppose to the contrary that (w.l.o.g.) $\min \left\{r, c_{i}\right\} \leq$ $\frac{r-\left(\delta_{i}-\delta_{j}\right)}{2}$ but $x_{i}^{*}<\min \left\{r, c_{i}\right\}$. Hence, by efficiency, $x_{j}^{*}=r-x_{i}^{*}>0$.

We consider two cases:

Case 1: $r \leq c_{i}$. In this case $r \leq \frac{r-\left(\delta_{i}-\delta_{j}\right)}{2}$ or

$$
r+\delta_{i} \leq \delta_{j} \text { and so } \delta_{j} \geq \delta_{i}
$$

Moreover, since $x^{*}=G E A(r, c, \delta)$ and $x_{i}^{*}<c_{i}$, we claim $x_{i}^{*}=\min \left\{c_{i},(\lambda-\right.$ $\left.\left.\delta_{i}\right)_{+}\right\}=\left(\lambda-\delta_{i}\right)_{+}=\lambda-\delta_{i}$; otherwise $0>\lambda-\delta_{i} \geq \lambda-\delta_{j}$, where the last inequality follows from (7), and then $x_{j}^{*}=0$ which implies a contradiction.

On the other hand, since $x^{*}=G E A(r, c, \delta)$ and $x_{j}^{*}>0$, we get $0<x_{j}^{*}=$ $\min \left\{c_{j},\left(\lambda-\delta_{j}\right)_{+}\right\}=\min \left\{c_{j}, \lambda-\delta_{j}\right\} \leq \lambda-\delta_{j}$. However, if $\lambda-\delta_{j}>0$ we would have that, by (7), $\lambda>\delta_{j} \geq r+\delta_{i}$ and so $r<\lambda-\delta_{i}=x_{i}^{*}$ which is infeasible.

Case 2: $r>c_{i}$. In this case

$$
c_{i} \leq \frac{r-\left(\delta_{i}-\delta_{j}\right)}{2} .
$$


Since we are assuming that $x_{i}^{*}<c_{i}<r$ and by definition of the $G E A$ rule, we get $x_{i}^{*}=\min \left\{c_{i},\left(\lambda-\delta_{i}\right)_{+}\right\}=\left(\lambda-\delta_{i}\right)_{+}$. If $\lambda-\delta_{i} \geq 0$ we have $r=x_{i}^{*}+x_{j}^{*}=\lambda-\delta_{i}+x_{j}^{*} \leq \lambda-\delta_{i}+\lambda-\delta_{j}$, where the last inequality follows from $0<x_{j}^{*}=\min \left\{c_{j},\left(\lambda-\delta_{j}\right)_{+}\right\} \leq \lambda-\delta_{j}$. Henceforth $r+\delta_{j} \leq 2 \lambda-\delta_{i}$ and so, substituting in (8), $c_{i} \leq \lambda-\delta_{i}$ which implies that $x_{i}^{*}=c_{i}$ contradicting our hypothesis. On the other hand, if $\lambda-\delta_{i}<0$ we have that $x_{i}^{*}=0$ and $r=x_{j}^{*} \leq \lambda-\delta_{j}$. Henceforth $r+\delta_{j} \leq \lambda$ and so, substituting in (8), $c_{i} \leq \frac{\lambda-\delta_{i}}{2}<0$ which is a contradiction.

We conclude that the $G E A$ rule satisfies compensated exemption.

Proposition 5. The GEA rule is consistent.

Proof. Notice that in Proposition 1 we characterize the GEA rule by making payoff bilateral comparisons. Then, it is straightforward that if we reconsider the problem within a subgroup of agents, then the solution must fulfill the same bilateral comparisons and so the solution does not change.

The next theorem characterizes the $G E A$ rule by means of the properties we have presented.

Theorem 1. The GEA rule is the unique rule that satisfies path-independence, compensated exemption and consistency.

Proof. The GEA rule satisfies consistency (Proposition 5), path-independence (Proposition 3) and compensated exemption (Proposition 4). Let us check that it is unique. Let $F$ be a rule satisfying these properties. If $|N|=1$, it is straightforward. Consider now the two-person case $N=\{1,2\}$ and $(r, c, \delta) \in \mathcal{R}^{\{1,2\}}$. Let us denote $x^{*}=\left(x_{1}^{*}, x_{2}^{*}\right)=F(r, c, \delta)$. Let us suppose that (w.l.o.g.) $\delta_{1} \leq \delta_{2}$. We consider three cases:

Case 1: $r \leq \delta_{2}-\delta_{1}$. Then,

$$
\min \left\{r, c_{1}\right\} \leq r=\frac{r}{2}+\frac{r}{2} \leq \frac{r-\left(\delta_{1}-\delta_{2}\right)}{2} .
$$

Hence, by compensated exemption, we have that $x_{1}^{*}=\min \left\{r, c_{1}\right\}$ and $x_{2}^{*}=$ $\left(r-c_{1}\right)_{+}$and so it is uniquely determined. 
Case 2: $r>\delta_{2}-\delta_{1} \geq c_{1}$. Then,

$$
\min \left\{r, c_{1}\right\}=c_{1} \leq \delta_{2}-\delta_{1}=\frac{\delta_{2}-\delta_{1}}{2}+\frac{\delta_{2}-\delta_{1}}{2}<\frac{r-\left(\delta_{1}-\delta_{2}\right)}{2} .
$$

Hence, by compensated exemption, we have that $x_{1}^{*}=\min \left\{r, c_{1}\right\}=c_{1}$ and $x_{2}^{*}=r-c_{1}$ and so it is uniquely determined.

Case 3: $r>\delta_{2}-\delta_{1}$ and $c_{1}>\delta_{2}-\delta_{1}$. We consider two subcases:

Subcase 3.a: $c_{1}+\delta_{1}=c_{2}+\delta_{2}$. Since $r>\delta_{2}-\delta_{1}$ and by Lemma 1 (see Appendix) we have that $x_{1}^{*}+\delta_{1}=x_{2}^{*}+\delta_{2}$ and so, taking into account that $x_{1}^{*}+x_{2}^{*}=r$, it is uniquely determined.

Subcase 3.b: $c_{1}+\delta_{1} \neq c_{2}+\delta_{2}$. First, if for agent $1 \min \left\{r, c_{1}\right\} \leq \frac{r-\left(\delta_{1}-\delta_{2}\right)}{2}$, then, by compensated exemption, $x_{1}^{*}=\min \left\{r, c_{1}\right\}$ and $x_{2}^{*}=\left(r-c_{1}\right)_{+}$and so it is uniquely determined. Similarly for agent 2 , if $\min \left\{r, c_{2}\right\} \leq \frac{r-\left(\delta_{2}-\delta_{1}\right)}{2}$, then, by compensated exemption, $x_{2}^{*}=\min \left\{r, c_{2}\right\}$ and $x_{1}^{*}=\left(r-c_{2}\right)_{+}$and so it is uniquely determined. Otherwise,

$$
\min \left\{r, c_{i}\right\}+\delta_{i}>\frac{r+\delta_{1}+\delta_{2}}{2} \text { for all } i \in\{1,2\} .
$$

Let us suppose that

$$
c_{i}+\delta_{i}<c_{j}+\delta_{j}, \text { where } i, j \in\{1,2\} \text { with } i \neq j .
$$

Now we claim that for $r^{\prime}=2 c_{i}+\delta_{i}-\delta_{j}$, we have that $x^{\prime}=F\left(r^{\prime}, c, \delta\right)$ is such that $x_{i}^{\prime}=c_{i}$ and $x_{j}^{\prime}=c_{i}+\delta_{i}-\delta_{j}$. To check it, first notice that, by (10), $r^{\prime}<c_{i}+c_{j}$. Moreover, notice that $c_{i}+\delta_{i}-\delta_{j} \geq 0$; otherwise $c_{i}<\delta_{j}-\delta_{i}$ and if $i=1$, we would get a contradiction with the hypothesis of Case 3, and if $i=2, c_{2}<\delta_{1}-\delta_{2} \leq 0$ and we would reach a contradiction, where the second inequality follows from the assumption $\delta_{1} \leq \delta_{2}$. Now, notice that we can apply compensated exemption to the agent $i$ in the problem $\left(r^{\prime}, c, \delta\right)$, since $c_{i}+\delta_{i}-\delta_{j} \geq 0$, and so

$$
\min \left\{r^{\prime}, c_{i}\right\}+\delta_{i}=\min \left\{2 c_{i}+\delta_{i}-\delta_{j}, c_{i}\right\}+\delta_{i}=c_{i}+\delta_{i}=\frac{r^{\prime}+\delta_{i}+\delta_{j}}{2} .
$$

Hence, by compensated exemption, we have that $x_{i}^{\prime}=c_{i}$. By efficiency, $x_{j}^{\prime}=r^{\prime}-x_{i}^{\prime}=c_{i}+\delta_{i}-\delta_{j}$, and the proof of the claim is done. 
On the other hand, let us remark that $r^{\prime}=2 c_{i}+\delta_{i}-\delta_{j} \geq 2 \min \left\{r, c_{i}\right\}+$ $\delta_{i}-\delta_{j}>r$, where the last inequality follows from (9). Therefore, by pathindependence, we obtain

$$
F(r, c, \delta)=F\left(r, F\left(r^{\prime}, c, \delta\right), \delta\right)=F\left(r, x^{\prime}, \delta\right)
$$

Finally, notice that we can apply Lemma 1 to the problem $\left(r, x^{\prime}, \delta\right)$ since $x_{j}^{\prime}+\delta_{j}=c_{i}+\delta_{i}=x_{i}^{\prime}+\delta_{i}$ and $r>\delta_{2}-\delta_{1}$, where the inequality is the hypothesis of Case 3. Then we get

$$
F_{i}(r, c, \delta)+\delta_{i}=F_{i}\left(r, x^{\prime}, \delta\right)+\delta_{i}=F_{j}\left(r, x^{\prime}, \delta\right)+\delta_{j}=F_{j}(r, c, \delta)+\delta_{j},
$$

where the first and the last equalities follow from path-independence and the remainder equality follows from Lemma 1 . Hence it is uniquely determined. Then we conclude that, for the two-person case, the $G E A$ rule is the unique rule that satisfies path-independence and compensated exemption.

Let $|N| \geq 3$ and suppose that $F$ and $F^{\prime}$ satisfies the three properties but $F \neq F^{\prime}$. Hence, there exists $(r, c, \delta) \in \mathcal{R}^{N}$ such that $x=F(r, c, \delta) \neq$ $F^{\prime}(r, c, \delta)=x^{\prime}$. This means that there exists $i, j \in N$ such that $x_{i}>x_{i}^{\prime}$, $x_{j}<x_{j}^{\prime}$ and $x_{i}+x_{j} \leq x_{i}^{\prime}+x_{j}^{\prime}$. However, since $F$ and $F^{\prime}$ are consistent,

$$
\begin{aligned}
& \left(x_{i}, x_{j}\right)=F\left(r-\sum_{k \in N \backslash\{i, j\}} x_{k},\left(c_{i}, c_{j}\right),\left(\delta_{i}, \delta_{j}\right)\right) \text { and } \\
& \left(x_{i}^{\prime}, x_{j}^{\prime}\right)=F^{\prime}\left(r-\sum_{k \in N \backslash\{i, j\}} x_{k}^{\prime},\left(c_{i}, c_{j}\right),\left(\delta_{i}, \delta_{j}\right)\right) .
\end{aligned}
$$

Since path-independence implies resource monotonicity (see page 13) and $F=F^{\prime}$ for the two-person case, we have that

$$
\begin{aligned}
\left(x_{i}^{\prime}, x_{j}^{\prime}\right) & =F^{\prime}\left(x_{i}^{\prime}+x_{j}^{\prime},\left(c_{i}, c_{j}\right)\left(\delta_{i}, \delta_{j}\right)\right)=F\left(x_{i}^{\prime}+x_{j}^{\prime},\left(c_{i}, c_{j}\right)\left(\delta_{i}, \delta_{j}\right)\right) \\
& \geq F\left(x_{i}+x_{j},\left(c_{i}, c_{j}\right)\left(\delta_{i}, \delta_{j}\right)\right)=\left(x_{i}, x_{j}\right) .
\end{aligned}
$$

Hence we reach a contradiction with $x_{i}>x_{i}^{\prime}$. We conclude that the rule is unique and $F=G E A$.

The properties that characterize the $G E A$ rule are independent as the reader may check in Examples 3, 4 and 5 in the Appendix. 
In what follows, we characterize the $G E L$ rule using the fact that the $G E A$ and GEL are dual rules (see Proposition 2). The properties that characterize the $G E L$ rule are the corresponding dual ones that characterize the $G E A$ rule.

Definition 8. $\mathcal{P}^{*}$ is the dual property of $\mathcal{P}$ if for every rule $F$ it is true that $F$ satisfies $\mathcal{P}$ if and only if its dual rule $F^{*}$ satisfies $\mathcal{P}^{*}$.

The dual property of compensated exemption is compensated exclusion (see Proposition 6 in Appendix).

Property 4. A generalized rationing rule $F$ satisfies compensated exclusion if for any two-person rationing problem $(r, c, \delta) \in \mathcal{R}^{\{i, j\}}$ it holds that

$$
\text { if } \min \left\{L, c_{i}\right\} \leq \frac{L-\left(\delta_{j}-\delta_{i}\right)}{2} \Longrightarrow F_{i}(r, c, \delta)=\left(r-c_{j}\right)_{+} \text {. }
$$

Parallel to standard rationing problems (without payoff thresholds), the dual property of path-independence is composition (see Proposition 7 in Appendix).

Property 5. A generalized rationing rule $F$ satisfies composition if for all $(r, c, \delta) \in \mathcal{R}^{N}$ and all $r_{1}, r_{2} \in \mathbb{R}_{+}$such that $r_{1}+r_{2}=r$, it holds

$$
F(r, c, \delta)=F\left(r_{1}, c, \delta\right)+F\left(r_{2}, c-F\left(r_{1}, c, \delta\right), \delta\right) .
$$

Before giving the characterization result for the $G E L$ rule, let us mention that Herrero and Villar connect the properties that characterize a rule for a standard rationing problem with the properties that characterize the corresponding dual rule.

"Theorem 0 (Herrero and Villar, 2001). If a rule $F$ is characterized by a set of independent properties $\mathcal{P}=\left\{\mathcal{P}_{1}, \mathcal{P}_{2}, \ldots, \mathcal{P}_{k}\right\}$ and if for any $\mathcal{P}_{i}$ there exists a dual property $\mathcal{P}_{i}^{*}$, then the dual rule $F^{*}$ is characterized by the corresponding set of dual properties $\mathcal{P}^{*}=\left\{\mathcal{P}_{1}^{*}, \mathcal{P}_{2}^{*}, \ldots, \mathcal{P}_{k}^{*}\right\}$. Moreover, the properties in $\mathcal{P}^{*}$ are also independent". 
This result can be extended directly to the case of our generalized framework. This allows to characterize the $G E L$ rule by the corresponding dual properties that characterize the $G E A$ rule.

Theorem 2. The GEL rule is the unique rule that satisfies composition, compensated exclusion and consistency.

Proof. We know that GEA and GEL are dual rules from each other (Proposition 2). Moreover, compensated exclusion is the dual property of compensated exemption (Proposition 6), composition is the dual property of pathindependence (Proposition 7) and consistency is dual of itself. Therefore, the result follows from Theorem 0 of Herrero and Villar (2001).

\section{Conclusions}

We have presented an extension of the standard model of rationing situations. The aim of this extension is to take into account ex-ante inequalities of agents (different from claims) involved in the rationing process and try to compensate these inequalities. Two of the most outstanding rules (equal awards and equal losses) have been generalized and characterized within this new framework.

Two final remarks can be done as a future research work. Firstly, in the standard rationing framework, there are two important solutions that we have not analysed yet: the Talmudic rule and the proportional rule. With respect to the Talmudic rule, notice that in the standard framework the Talmudic is a self-dual rule and it is based essentially on the $C E A$ and the $C E L$ rule. An extension to the case of rationing problems with payoff thresholds and an analysis of self-duality might be interesting. With respect to the proportional solution (another self-dual solution), it is not so clear that the extension to the payoff thresholds framework should also maintain the self-duality property.

Secondly, the consideration of payoff thresholds can be attributed not only to priorities, past allocations or debts, but also to the fact that the same set of 
agents might be involved in simultaneous or parallel rationing situations and so we have to take into account what agents receive in a particular rationing situation to distribute the resource in the remaining rationing situations. The problem becomes not of a single-issue but of a multi-issue rationing nature. A global approach to the overall rationing is needed. Payoff thresholds in each particular single-issue rationing will not be exogenous to the model, but endogenous in a global multi-issue rationing situation. The definition of rules and extensions of the $G E A$ rule and the $G E L$ rule needs to be afforded.

\section{References}

[1] Aumann, R., \& Maschler, M. (1985). Game theoretic analysis of a bankruptcy problem from the Talmud. Journal of Economic Theory 36, 195-213.

[2] Herrero, C., \& Villar, A. (2001). The three musketeers: four classical solutions to bankruptcy problems. Mathematical Social Sciences 39, 307-328.

[3] Hougaard, J.L., Moreno-Ternero, J., \& Østerdal, L.P. (2012). A unifying framework for the problem of adjudicating conflicting claims. Journal of Mathematical Economics 48, 107-114.

[4] Hougaard, J.L., Moreno-Ternero, J., \& Østerdal, L.P. (2013a). Rationing in the presence of baselines. Social Choice and Welfare 40, 1047-1066.

[5] Hougaard, J.L., Moreno-Ternero, J., \& Østerdal, L.P. (2013b). Rationing with baselines: the composition extension operator. Annals of Operations Research. DOI 10.1007/s10479-013-1471-8.

[6] Kaminski, M. (2000). Hydraulic rationing. Mathematical Social Sciences 40, 131-155. 
[7] Moulin, H. (2000). Priority rules and other asymmetric rationing methods. Econometrica 68, 643-684.

[8] O'Neill, B. (1982). A problem of rights arbitration from the Talmud. Mathematical Social Sciences 2, 345-371.

[9] Pulido, M., Borm, P., Hendrickx, R., Llorca, N., \& Sánchez-Soriano, J. (2008). Compromise Solutions for Bankruptcy Situations with references. Annals of Operations Research 158, 133-141.

[10] Pulido, M., Sánchez-Soriano, J. \& Llorca, N. (2002). Game theory techniques for university management: an extended bankruptcy model. Annals of Operations Research 109, 129-142.

[11] Thomson, W. (2003). Axiomatic and game-theoretic analysis of bankruptcy and taxation problems: a survey. Mathematical Social Sciences 45, 249-297.

\section{Appendix}

Lemma 1. If a generalized rationing rule $F$ satisfies compensated exemption and path-independence then, for any $(r, c, \delta) \in \mathcal{R}^{\{1,2\}}$ it holds that

$$
\text { if } c_{1}+\delta_{1}=c_{2}+\delta_{2} \text { and } r>\left|\delta_{1}-\delta_{2}\right| \Longrightarrow F_{1}(r, c, \delta)+\delta_{1}=F_{2}(r, c, \delta)+\delta_{2} \text {. }
$$

Proof. Let $x^{*}=F(r, c, \delta)$. Consider $c_{1}+\delta_{1}=c_{2}+\delta_{2}$ and $r>\left|\delta_{1}-\delta_{2}\right|$, but let us suppose to the contrary that (w.l.o.g.)

$$
x_{1}^{*}+\delta_{1}<x_{2}^{*}+\delta_{2}
$$

By (11), notice that $x_{1}^{*}+\delta_{1}<\frac{x_{1}^{*}+\delta_{1}+x_{2}^{*}+\delta_{2}}{2}=\frac{r+\delta_{1}+\delta_{2}}{2}$ and so $x_{1}^{*}<\frac{r+\delta_{2}-\delta_{1}}{2}$.

CLAIM. There exists $r^{\prime}>r$ such that $F_{1}\left(r^{\prime}, c, \delta\right)=\frac{r+\delta_{2}-\delta_{1}}{2}$.

Proof. Notice that $\frac{r+\delta_{2}-\delta_{1}}{2}>0$ since $x_{1}^{*} \geq 0$. Notice also that $\frac{r+\delta_{2}-\delta_{1}}{2} \leq c_{1}$ since $c_{1}+\delta_{1}=c_{2}+\delta_{2}$. Since $F$ satisfies path-independence it also satisfies resource monotonicity. Hence, $F$ is a continuous and increasing function of $r$. 
Therefore, by continuity, since $F_{1}(0, c, \delta)=0, F_{1}\left(c_{1}+c_{2}, c, \delta\right)=c_{1}$ and $F$ is an increasing function in $r$, there will exist $r^{\prime}$ such that $F_{1}\left(r^{\prime}, c, \delta\right)=\frac{r+\delta_{2}-\delta_{1}}{2}$ and we are done.

Let us denote $x^{\prime}=F\left(r^{\prime}, c, \delta\right)$. Notice that $\min \left\{r, x_{1}^{\prime}\right\} \leq x_{1}^{\prime}=\frac{r-\left(\delta_{1}-\delta_{2}\right)}{2}$ which implies, by compensated exemption applied to the problem $\left(r, x^{\prime}, \delta\right)$, that $F_{1}\left(r, x^{\prime}, \delta\right)=\min \left\{r, x_{1}^{\prime}\right\}=\min \left\{r, \frac{r+\delta_{2}-\delta_{1}}{2}\right\}=\frac{r+\delta_{2}-\delta_{1}}{2}$, where the last equality follows from $r>\left|\delta_{2}-\delta_{1}\right|$. Finally, by path-independence, we obtain

$$
F(r, c, \delta)=F\left(r, F\left(r^{\prime}, c, \delta\right), \delta\right)=\left(\frac{r+\delta_{2}-\delta_{1}}{2}, \frac{r+\delta_{1}-\delta_{2}}{2}\right)
$$

and we conclude that $x_{1}^{*}+\delta_{1}=\frac{r+\delta_{1}+\delta_{2}}{2}=x_{2}^{*}+\delta_{2}$.

Example 3. Let $F$ be a generalized rationing rule such that, for all $(r, c, \delta)$,

$$
F(r, c, \delta)=G E A(r, c, \overrightarrow{0})
$$

This rule satisfies consistency and path-independence but does not verify compensated exemption.

Example 4. Let $(r, c, \delta) \in \mathcal{R}^{N}$ and let us denote by $\widehat{c}_{i}=\min \left\{r, c_{i}\right\}$ the truncated claim of agent $i \in N$. Up to reordering of agents, there exist $k_{1}, k_{2}, \ldots, k_{m}$ such that $k_{1}+k_{2}+\ldots+k_{m}=n$ and

$$
\begin{aligned}
\widehat{c}_{1}+\delta_{1} & =\widehat{c}_{2}+\delta_{2}=\ldots=\widehat{c}_{k_{1}}+\delta_{k_{1}} \\
& <\widehat{c}_{k_{1}+1}+\delta_{k_{1}+1}=\widehat{c}_{k_{1}+2}+\delta_{k_{1}+2}=\ldots=\widehat{c}_{k_{1}+k_{2}}+\delta_{k_{1}+k_{2}} \\
& <\widehat{c}_{k_{1}+k_{2}+1}+\delta_{k_{1}+k_{2}+1}=\ldots=\widehat{c}_{k_{1}+k_{2}+k_{3}}+\delta_{k_{1}+k_{2}+k_{3}} \\
& \vdots \\
& <\widehat{c}_{k_{1}+\ldots+k_{m-1}+1}+\delta_{k_{1}+\ldots+k_{m-1}+1}=\ldots=\widehat{c}_{k_{1}+\ldots+k_{m}}+\delta_{k_{1}+\ldots+k_{m}}
\end{aligned}
$$

Notice we have grouped the agents in $m$ groups according to the value $\widehat{c}_{i}+$ $\delta_{i}$, where this value is constant within groups and strictly increasing across groups. Let us denote each group by $N_{1}=\left\{i \in N: 1 \leq i \leq k_{1}\right\}$ and $N_{t}=\left\{i \in N: k_{1}+\ldots+k_{t-1}+1 \leq i \leq k_{1}+\ldots+k_{t}\right\}$, for all $t \in\{2, \ldots, m\}$. 
Then, can define recursively an allocation rule by assigning payoffs to the members of each group as follows.

Step 1 (group $N_{1}$ ):

If $\sum_{i \in N_{1}} c_{i} \geq r$ then $x_{i}=G E A_{i}\left(r, c_{\mid N_{1}}, \delta_{\mid N_{1}}\right)$, for all $i \in N_{1}$, and $x_{i}=0$, otherwise. Stop.

If not, $\sum_{i \in N_{1}} c_{i}<r$, we assign $x_{i}=c_{i}$, for all $i \in N_{1}$ and we go to the next step.

Step t $\left(2 \leq t \leq m\right.$, groups $N_{2}$ to $\left.N_{m}\right)$ :

$$
\text { If } \sum_{i \in N_{t}} c_{i} \geq r-\sum_{\substack{i \in N_{j} \\ j=1, \ldots, t-1}} c_{i} \text { then } x_{i}=G E A_{i}\left(r-\sum_{\substack{i \in N_{j} \\ j=1, \ldots, t-1}} c_{i}, c_{\mid N_{t}}, \delta_{\mid N_{t}}\right) \text {, }
$$

for all $i \in N_{t}$, and $x_{i}=0$, for all $i \in N_{k}$ with $k=t+1, t+2, \ldots, m$. Stop.

If not, $\sum_{i \in N_{t}} c_{i}<r-\sum_{\substack{i \in N_{j} \\ j=1, \ldots, t-1}} c_{i}$, we assign $x_{i}=c_{i}$, for all $i \in N_{t}$ and we go to the next step.

This rule satisfies consistency and compensated exemption but does not verify path-independence.

Example 5. Let $|N| \geq 3$ and define $N=N_{1} \cup N_{2}=\{1,2, \ldots, n\}$, where $N_{1}=\{1,2\}$ and $N_{2}=N \backslash N_{1}$. Let $C_{N_{1}}=c_{1}+c_{2}, C_{N_{2}}=\sum_{i \in N_{2}} c_{i}, \Delta_{N_{1}}=$ $\delta_{1}+\delta_{2}$, and $\Delta_{N_{2}}=\sum_{i \in N_{2}} \delta_{i}$. Next, let us denote by $z=\left(z_{1}, z_{2}\right)$ the allocation obtained by applying the GEA rule to the two-subgroup problem; that is

$$
z=\left(z_{1}, z_{2}\right)=\operatorname{GEA}\left(r,\left(C_{N_{1}}, C_{N_{2}}\right),\left(\Delta_{N_{1}}, \Delta_{N_{2}}\right)\right) .
$$

Then, define the rule $F$ as follows: if $|N| \leq 2, F(r, c, \delta)=G E A(r, c, \delta)$; if $|N| \geq 3$

$$
F_{i}(r, c, \delta)= \begin{cases}G E A_{i}\left(z_{1},\left(c_{1}, c_{2}\right),\left(\delta_{1}, \delta_{2}\right)\right) & \text { if } i \in N_{1}, \\ G E A_{i}\left(z_{2},\left(c_{i}\right)_{i \in N_{2}},\left(\delta_{i}\right)_{i \in N_{2}}\right) & \text { if } i \in N_{2} .\end{cases}
$$

This rule $F$ satisfies compensated exemption and path-independence but it is not consistent. 
Proposition 6. Compensated exemption and compensated exclusion are dual properties.

Proof. Let $(r, c, \delta) \in \mathcal{R}^{\{1,2\}}$ be a two-person rationing problem with payoff thresholds and let us suppose that $F$ and $F^{*}$ are dual rules, that is $F^{*}(r, c, \delta)=c-F(L, c,-\delta)$. Hence, we claim that if $F$ satisfies compensated exemption then $F^{*}$ satisfies compensated exclusion. To check it, suppose (w.l.o.g.) that for the problem $(r, c, \delta)$, we have

$$
\min \left\{L, c_{1}\right\} \leq \frac{L-\left(\delta_{2}-\delta_{1}\right)}{2} .
$$

Notice that (12) is the same condition that we use in the definition of compensated exemption when we apply the rule $F$ to the problem $(L, c,-\delta)$. Hence, since $F$ satisfies compensated exemption and by (12), we have

$$
\begin{aligned}
F_{1}^{*}(r, c, \delta) & =c_{1}-F_{1}(L, c,-\delta)=c_{1}-\min \left\{c_{1}, L\right\}=\max \left\{0, c_{1}-L\right\} \\
& =\max \left\{0, c_{1}-\left(c_{1}+c_{2}-r\right)\right\}=\left(r-c_{2}\right)_{+} .
\end{aligned}
$$

We conclude that $F^{*}$ satisfies compensated exclusion.

Similarly, we claim that if $F$ satisfies compensated exclusion then $F^{*}$ satisfies compensated exemption. Let us suppose (w.l.o.g.) that for the problem $(r, c, \delta)$, we have

$$
\min \left\{r, c_{1}\right\} \leq \frac{r-\left(\delta_{1}-\delta_{2}\right)}{2} .
$$

Notice that (13) is the same condition that we use in the definition of compensated exclusion when we apply the rule $F$ to the problem $(L, c,-\delta)$. Hence, since $F$ satisfies compensated exclusion we have that

$$
\begin{aligned}
F_{1}^{*}(r, c, \delta) & =c_{1}-F_{1}(L, c,-\delta)=c_{1}-\left(L-c_{2}\right)_{+} \\
& =c_{1}-\max \left\{0, L-c_{2}\right\}=\min \left\{c_{1}, c_{1}+c_{2}-L\right\} \\
& =\min \left\{c_{1}, r\right\} .
\end{aligned}
$$

We conclude that $F^{*}$ satisfies compensated exemption. 
Proposition 7. Path-independence and composition are dual properties.

Proof. Let us suppose that $F$ and $F^{*}$ are dual rules. Then, by definition $F^{*}(r, c, \delta)=c-F(L, c,-\delta)$. We claim that if $F$ satisfies composition then $F^{*}$ satisfies path-independence. To check it, let $r \geq r_{1} \geq 0$ and define $r_{2}=r-r_{1}$. Moreover, let $L_{1}=\sum_{i \in N} c_{i}-r_{1}$. Hence,

$$
L=\sum_{i \in N} c_{i}-r=L_{1}-r_{2}, \text { and so } L_{1} \geq L .
$$

On one hand, we have

$$
\begin{aligned}
F^{*}\left(r_{1}, c, \delta\right) & =c-F\left(L_{1}, c,-\delta\right) \\
& =c-\left(F(L, c,-\delta)+F\left(r_{2}, c-F(L, c,-\delta),-\delta\right)\right) \\
& =F^{*}(r, c, \delta)-F\left(r_{2}, c-F(L, c,-\delta),-\delta\right),
\end{aligned}
$$

where the first and the last equality follows from definition of dual rule and the second one follows from the composition property of $F$ and (14).

On the other hand, by definition of dual rule, we have

$$
\begin{aligned}
F^{*}\left(r_{1}, F^{*}(r, c, \delta), \delta\right) & =F^{*}(r, c, \delta)-F\left(r-r_{1}, F^{*}(r, c, \delta),-\delta\right) \\
& =F^{*}(r, c, \delta)-F\left(r_{2}, c-F(L, c,-\delta),-\delta\right) .
\end{aligned}
$$

Therefore, since (15) and (16), $F^{*}$ satisfies path-independence.

Similarly, we claim that if $F$ satisfies path-independence then $F^{*}$ satisfies composition. To check it, let $r_{1}+r_{2}=r$, where $r_{1}, r_{2} \in \mathbb{R}_{+}$and $L_{1}=$ $\sum_{i \in N} c_{i}-r_{1}$. Notice that $L_{1} \geq L$. By path-independence and by definition of dual rule, we have

$$
\begin{aligned}
F(L, c,-\delta) & =F\left(L, F\left(L_{1}, c,-\delta\right),-\delta\right) \\
& =F\left(L_{1}, c,-\delta\right)-F^{*}\left(r_{2}, F\left(L_{1}, c,-\delta\right), \delta\right) .
\end{aligned}
$$

Then, by definition of dual rule and by (17), we have

$$
\begin{aligned}
F^{*}(r, c, \delta) & =c-F(L, c,-\delta) \\
& =c-\left(F\left(L_{1}, c,-\delta\right)-F^{*}\left(r_{2}, F\left(L_{1}, c,-\delta\right), \delta\right)\right) \\
& =F^{*}\left(r_{1}, c, \delta\right)+F^{*}\left(r_{2}, F\left(L_{1}, c,-\delta\right), \delta\right) \\
& =F^{*}\left(r_{1}, c, \delta\right)+F^{*}\left(r_{2}, c-F^{*}\left(r_{1}, c, \delta\right), \delta\right) .
\end{aligned}
$$


Therefore $F^{*}$ satisfies composition. 